WHOI-78-8

\title{
SHAPES OF DEEP DENSITY-DEPTH CURVES
}

by

Bruce A. Warren

WOODS HOLE OCEANOGRAPHIC INSTITUTION

Woods Hole, Massachusetts 02543

April 1978

TECHNICAL REPORT

Prepared for the Office of Naval Research under Contract N00014-74-C-0262; NR 083-004.

Reproduction in whole or in part is permitted for any purpose of the United States Govermment. In citing this report in a bibliography, the reference given should be to the "Jourmal of Physical Oceanography, Vol. 7, No. 3, May 1977".

Approved for public release; distribution unlimited.

Approved for Distribution: Valentine Worthington, Chairman Department of Physical Oceanography 

Reprinted from Journal of Physical Oceanography, Vol. 7, No. 3, May 1977

American Meteorological Society

Printed in U.S. A.

\section{Shapes of Deep Density-Depth Curves}

BRUCE A. WARREN 


\title{
Shapes of Deep Density-Depth Curves ${ }^{1}$
}

\author{
BRUCE A. WARREN \\ Woods Hole Oceanographic Institution, Woods Hole, Mass. 02543 \\ Manuscript received 28 September 1976, in revised form 24 January 1977)
}

\begin{abstract}
Deep density-depth curves often show faint curvature reversals, stability maxima, etc. Relationships between these features and the field of motion are explored here, in reference to the South Pacific, for interior regions and western boundary currents. For the former, an assumed balance between vertical advection and vertical diffusion of density implies downward movement where there is positive curvature in the density-depth curve, in association with equatorward flow of the bottom water; it is argued that such flow is a likely feature of the interaction between a stratified deep western boundary current and a stratified interior. Illustrative (though somewhat unrealistic) analytical examples are given. In the western boundary region it is the difference between local and interior densities which seems to be related to details of the velocity distribution, but only in special (and unlikely) circumstances is there a correspondence across the boundary region between the shape of the perturbation density-depth curve and the motion field. Some comments are made concerning the implications for the interpretation of hydrographic data.
\end{abstract}

\section{Introduction}

Inasmuch as the thermal wind relation gives only the vertical shear of horizontal velocity, descriptive oceanographers must seek other techniques to gage the absolute sense of flow. Since enough current records of sufficient length to give stable means are in general prohibitively expensive to obtain, one commonly appeals - usually intuitively - to features of property distributions (e.g., oxygen minima) for guidance in fixing integration constants for geostrophic calculations. Among other things, the shapes of density-depth curves have invited interpretations in terms of horizontal flow. Thus Reid and Lonsdale (1974) point out a deep stability maximum in the tropical South Pacific, and they suggest, intuitively, that it represents the large-scale boundary between southward-moving, low-oxygen water above and northward-moving, high-oxygen water below.

In other South Pacific data, Craig et al. (1972) believe that they see a "front" at inflections in the densitydepth curves, and they regard this "front"-also intuitively - as the boundary between the water flowing southward (above) and that flowing northward (below). Not all readers are able to detect the frontal discontinuity that Craig et al. (1972) find, but the existence of curvature reversals in many of their illustrative densitydepth curves is beyond question. Undoubtedly these curvature reversals, as well as the deeper stability maxima of Reid and Lonsdale (1974), are related to

\footnotetext{
${ }^{1}$ Contribution No. 3871 from the Woods Hole Oceanographic Institution.
}

the field of motion, but some understanding of the physical relationship needs to be developed if they are to be used eventually in interpreting hydrographic data.

The features themselves are fairly marginal, and might perhaps be dismissed as noise at any individual station if they did not also appear at other stations over a broad region. Some illustrative curves of $\sigma_{\theta}$ against depth from the deep South Pacific are plotted in Fig. 1.2 Scorpio Station 151 was made in the deep westernboundary current, and the density-depth curve has an interval of positive curvature at $2200-3000 \mathrm{~m}$, with a stability maximum at $3000 \mathrm{~m}$. Scorpio Stations 48 and 132 are from the eastern part of the Southwest Pacific Basin, an "interior" region, and these density-depth curves have intervals of much fainter positive curvature, centered near $3200 \mathrm{~m}$ (questionable perhaps at Station 48 ), with stability maxima below. The features are somewhat clearer at Kiwi Station 67, which was also made in the eastern part of the basin, but at a more northern latitude where the basin is much narrower, and the meridional flow is necessarily more confined; it may not be a good example of an "interior" station, as contrasted with a "western-boundary" station.

The dominant effects governing the deep density distribution no doubt vary from place to place in the ocean; at the very least the density balance in the interior of the ocean is probably different from that in the western-boundary region (Warren, 1976). In

\footnotetext{
${ }^{2}$ It is immaterial which of the various density parameters is used to illustrate the features; they appear equally well (or poorly) in plots of $\sigma_{t}$ or $\sigma_{4}$, for example.
} 


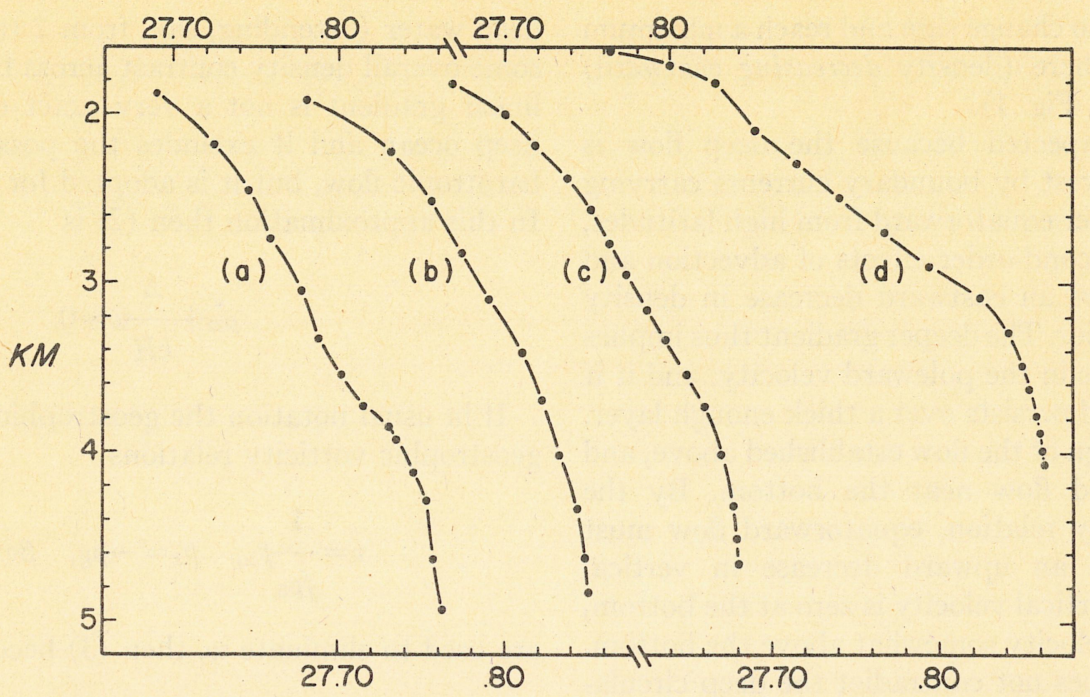

FIg. 1. Observed depth variations of $\sigma_{\theta}$ at four stations in the deep South Pacific: (a) Kiwi $67,22^{\circ} 17^{\prime} \mathrm{S}, 163^{\circ} 08^{\prime} \mathrm{W}, 26$ October 1969 ; (b) Scorpio $48,43^{\circ} 13^{\prime} \mathrm{S}, 132^{\circ} 15^{\prime} \mathrm{W}$,

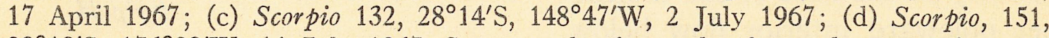
$28^{\circ} 18^{\prime} \mathrm{S}, 176^{\circ} 28^{\prime} \mathrm{W}, 14$ July 1967 . Separate density scales for each curve given alternately along top and bottom; dots indicate observed values. Station data on file at NODC.

attempting to rationalize such features as shown in Fig. 1, I shall therefore consider these two regions separately.

Some qualitative ideas will be discussed, and some analytical examples presented to illustrate them. In constructing the examples, however, I am forced to linearize to such an extent that they are somewhat removed from true oceanic conditions. Hence they are suitable mainly for depicting a kind of behavior, rather than for quantitative interpretation of real hydrographic data.

\section{Interior region}

The vertical coordinate $z$ is taken to be zero at the bottom, increasing upward, and coordinates $x$ and $y$ increasing eastward and poleward, respectively. The general equation governing the mean distribution of density $\rho$ is, with neglect of adiabatic temperature changes,

$$
k \rho_{z z}+K \nabla_{H}^{2} \rho=w \rho_{z}+\mathbf{V}_{H} \cdot \nabla_{H} \rho,
$$

where $w, \mathbf{V}_{H}$ are the vertical and horizontal components of velocity and $k, K$ are vertical and horizontal eddy diffusion coefficients. Let the vertical and horizontal scales of variation in $\rho$ be $D, L$; and let the typical magnitudes of the vertical and horizontal variations in $\rho$ over those scales be $\Delta_{V}$ and $\Delta_{H}$, with $\epsilon \equiv \Delta_{H} / \Delta_{V}$. If the interior flow is strictly geostrophic, $\left|\mathbf{V}_{H}\right| \sim(f w) /$ $(\beta D) \sim w R / D$, where $f, \beta$ have their usual meanings and $R$ is the radius of the earth. Then characteristic relative magnitudes of the four terms in (1) are, respectively,

$$
\begin{aligned}
& 1, \frac{K D^{2} \epsilon}{k L^{2}}, \quad \frac{w D}{k}, \frac{w D R \epsilon}{k L} \\
& 1,6 \times 10^{-2}, \quad 1, \quad 2 \times 10^{-1}
\end{aligned}
$$

with numerical estimates below based on values (in cgs units) appropriate to interior regions: $k \sim 1, K \sim 10^{7}$, $w \sim 10^{-5}, D \sim 10^{5}, L \sim R \sim 6 \times 10^{8}, \epsilon \sim 0.2$.

So long as $\epsilon \ll 1$, it is difficult to see how horizontal advection could have very much effect on the shape of the density-depth curve, except perhaps close to the bottom, where $w$ must go to zero and $\left|\mathbf{V}_{H}\right|$ may reach a maximum. Instead the dominant balance away from the bottom appears to be

$$
k \rho_{z z}=w \rho_{z} .
$$

If (2) is correct and $k$ is effectively constant, curvature reversals in $\rho(z)$ can only be accounted for by changes in sign of $w$; i.e., intervals of positive curvature must be associated with downward movement. Such movement does seem plausible for the very deep interior ocean. Suppose that in the central South Pacific the mean horizontal velocity is zero at $2000 \mathrm{~m}$, in the center of the oxygen-minimum layer-for which a very good account can be given in terms of Wyrtki's (1962) model of oxygen distribution in a layer with no horizontal flow. At these levels the density distribution is still faintly thermocline-like, the density increasing eastward; a gradient of such a sign implies a downward increase in the poleward component of flow. The density gradient is not observed to decay to zero at the 
bottom, but rather to change sign and reach a maximum of opposite sense there (density decreasing eastward) (e.g., Warren, 1976, Fig. 1).

This is to be expected because the deep flow is supplied from the west by boundary currents carrying relatively dense water equatorward from high latitudes, and by whatever second-order effects of advection and mixing, there follows an eastward decrease in density across the deep interior. The deeper gradient thus implies a downward decrease in the poleward velocity, and if it is strong enough and persists over a thick enough layer, it can reverse the sign of the flow established above, and require equatorward flow near the bottom. By the geostrophic vorticity relation, equatorward flow must be associated with an upward decrease in vertical velocity or, if the vertical velocity is zero at the bottom, with a downward velocity somewhat above the bottom.

This argument does not contradict the deep circulation theory of Stommel and Arons (1960), which assumes an upward velocity at the base of the thermocline, and infers a net poleward interior flow below. That theory was cast as a barotropic theory, but can be regarded in application to the real baroclinic ocean as a vertically integrated one, including the possibility of layers of equatorward flow within a deep flow that is poleward in vertical average. And, as suggested here, the interaction of a stratified boundary current with a stratified interior tends to require such a layer near the bottom.

To illustrate in a simple fashion the effect of a change in sign of horizontal density gradient on $\rho(z)$, consider a linearization of (2) by the common device of representing the density as $\rho=-z \Delta / H+\rho^{*}$ and assuming $\rho_{z}^{*} \ll \Delta / H$ (this condition to be specified more precisely below). Here $H$ will be regarded as the thickness of the

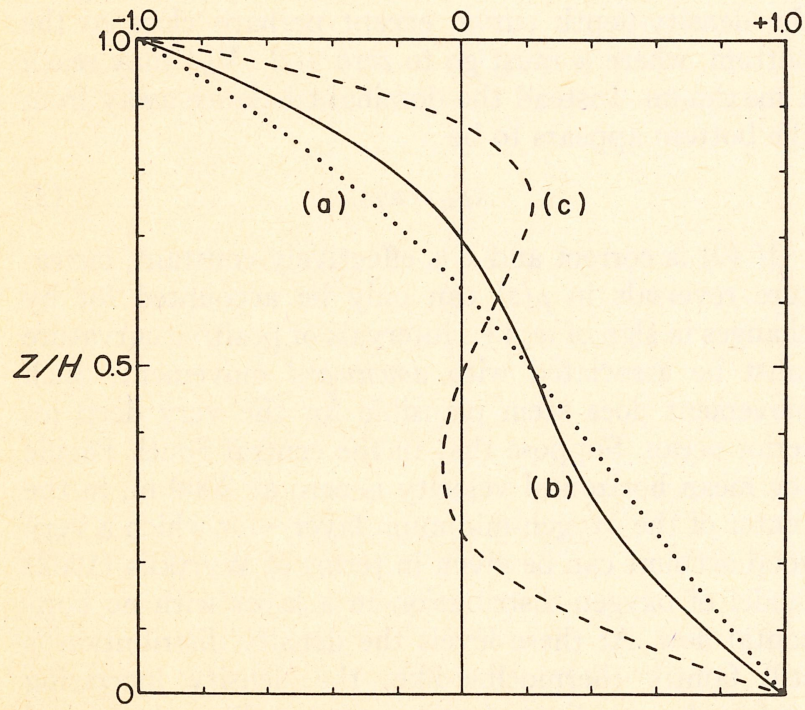

FIG. 2. Calculated interior solutions $\rho^{\prime}(z) / \sigma$ for (a) $d=1.5$ $\mathrm{km}$, (b) $d=1.0 \mathrm{~km}$, (c) $d=0.5 \mathrm{~km}$; all for $\rho_{B} / \sigma=-\rho_{T} / \sigma=1$. $H=3 \mathrm{~km}$. deep water (extending, say, from 2 to $5 \mathrm{~km}$ ), and $\Delta$ as some overall density contrast across that layer. A basic linear gradient is not a very exact description of the deep ocean and it excludes the possibility of a basic barotropic flow, but it is adopted for tractability later. In this approximation then (2) is

$$
\stackrel{*}{\rho_{z z}}+\frac{\Delta}{k H} w=0 .
$$

If in usual notation the geostrophic, hydrostatic and geostrophic vorticity relations

$$
v=\frac{1}{f \rho_{0}} p_{x}, \quad p_{z}=-\rho g, \quad \beta v=f w_{z}
$$

are used to eliminate $w$, then (3) becomes

$$
\rho_{z z z z}^{*}-\frac{g \beta \Delta}{k f^{2} \rho_{0} H} \rho_{x}^{*}=0 .
$$

The separable solution is of the form $\rho^{*}(x, z)=\rho^{\prime}(z)$ $\times \exp (-\alpha x)$, and fairly arbitrary horizontal distributions can be constructed from combinations of such solutions with different values of $\alpha$. For the separable solution (with positive $\alpha$ ),

$$
\rho_{\zeta \zeta \zeta \zeta}^{\prime}+4 \rho^{\prime}=0
$$

where $\zeta \equiv z / d$ and $d^{4} \equiv\left(4 H k f^{2} \rho_{0}\right) /(\Delta g \beta \alpha)$.

Clearly $\rho^{\prime}(z)$ has the vertical scale $d$; if its characteristic magnitude of variation is $\sigma$, then the condition for linearizing (2) to obtain (3) is $(\sigma / d)(H / \Delta) \ll 1$. It is not sufficient merely that $\sigma / \Delta \ll 1$, and since $d \sim 1 \mathrm{~km}$ for oceanic parameters, $(\sigma / d)(H / \Delta) \sim 1$ for the deep ocean. Calculations based on (3) are therefore not strictly realistic, and ought not to be taken as more than illustrating the general form of a likely effect.

The general solution of (5) is

$$
\rho^{\prime}(\zeta)=e^{\zeta}(A \cos \zeta+B \sin \zeta)+e^{-\zeta}(C \cos \zeta+D \sin \zeta),
$$

where $A, B, C, D$ are arbitrary constants. For boundary conditions, let $\rho^{\prime}$ be specified at the top and bottom of the deep water, let $w=0$ at the bottom, and let $v=0$ at the top (as suggested by the oxygen-minimum layer). Thus

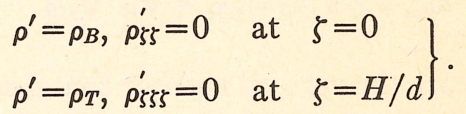

For $\rho_{B} / \sigma=+1, \rho_{T} / \sigma=-1$, solutions are shown in Fig. 2 for three values of $d: 0.5,1.0$ and $1.5 \mathrm{~km}$. For the reasonable oceanic values (cgs units) of $g=10^{3}, f=10^{-4}$, $\beta=2 \times 10^{-13}, k=1, \rho_{0}=1, \Delta=1.5 \times 10^{-4}$ and $H=3 \times 10^{5}$, these values of $d$ correspond to the values $\alpha^{-1}=156$, 2500 and $12657 \mathrm{~km}$, respectively. At the largest value of $d$, the change in sign of the curvature of $\rho^{\prime}(z)$ is only barely discernible and $\rho^{\prime}(z)$ is virtually linear in the 


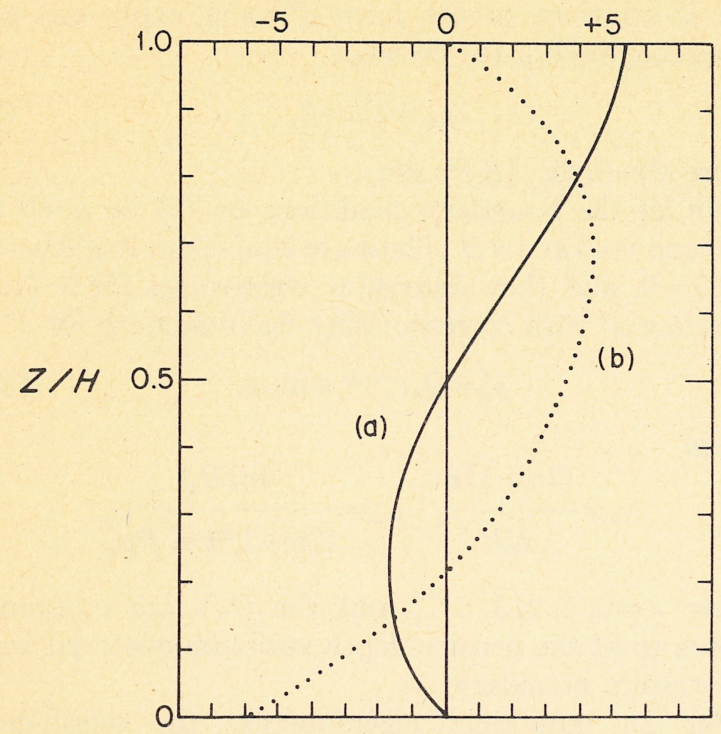

FIG. 3. Calculated distributions of (a) $10^{6} w(0, z)$ and (b) $200 v(0, z)$ for $d=1.0 \mathrm{~km}$ and $\rho_{B}=-\rho_{T}=10^{-5} \mathrm{~g} \mathrm{~cm}^{-3}$; velocity scale in $\mathrm{cm} \mathrm{s}^{-1} \cdot H=3 \mathrm{~km}$.

bottom kilometer; here there is only very slight downward movement in the deeper water, and correspondingly slight equatorward flow there. For the smaller values of $d$, the curves inflect clearly at the mid-depth, with negative curvature above, positive below, corresponding to intervals of upward and downward vertical velocities. For $d=0.5 \mathrm{~km}$, the curvature reversal is so sharp that $\rho^{\prime}$ has a maximum and minimum. The associated distributions of $w(0, z)$ and $v(0, z)$ for $d=1.0$ $\mathrm{km}$ and $\sigma=10^{-5} \mathrm{~g} \mathrm{~cm}^{-3}$ are illustrated in Fig. 3 .
The signs of $\rho_{B}, \rho_{T}$ are such that density increases eastward at the top of the deep water, and decreases eastward at the bottom, as in the deep South Pacific. For $\rho_{B}, \rho_{T}$ of opposite signs, the curves are simply the mirror images, because, according to the boundary conditions, the constants $A, B, C, D$ are each specified as linear combinations of $\rho_{B}, \rho_{T}$.

To indicate the effect of changes in $\rho_{B} / \rho_{T}$, curves of $\rho^{\prime}(z)$ are plotted in Fig. 4 for the single value $d=1.0 \mathrm{~km}$, but for $\rho_{T} / \sigma=-3, \rho_{B} / \sigma=+1$ and for $\rho_{T} / \sigma=-1$, $\rho_{B} / \sigma=+3$. The curve for $\rho_{T} / \sigma=-1, \rho_{B} / \sigma=+1$ is carried over from Fig. 2 for reference. It may be seen that the effect of increasing $\left|\rho_{T}\right|$ over $\left|\rho_{B}\right|$ is to depress the inflection point and to enhance the negative curvature while suppressing the positive curvature, whereas increasing $\left|\rho_{B}\right|$ over $\left|\rho_{T}\right|$ elevates the inflection point with corresponding opposite effects on the curvature. All these features make sense qualitatively in terms of the types of meridional flow to be expected from the thermal wind relation, together with the associated distributions of vertical velocity.

In all the examples shown, the curvature is positive throughout the lower part of the column, and the deep stability maximum occurs at the bottom itself. This circumstance is at variance with observation (Fig. 1), but is a necessary feature of any density distribution governed by (2), where the horizontal density gradient changes sign only once in the vertical. Negative curvature could be achieved in the near-bottom water (with a stability maximum above the bottom) by introducing another, deeper reversal in horizontal density gradient, but no such near-bottom reversal is observed in the South Pacific, nor does there seem any reason to expect one.

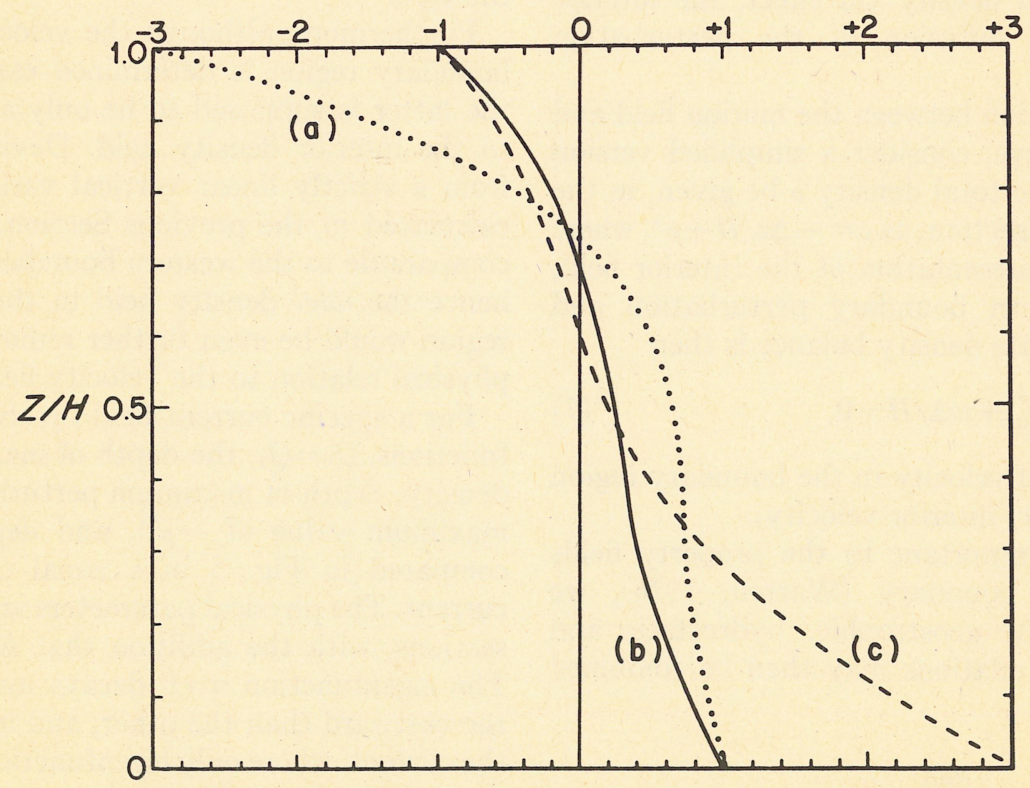

FIG. 4. Calculated interior solutions $\rho^{\prime}(z) / \sigma$ for $d=1.0 \mathrm{~km}, H=3 \mathrm{~km}$ and (a) $\rho_{T} / \sigma=-3, \rho_{B} / \sigma=+1$; (b) $\rho_{T} / \sigma=-1, \rho_{B} / \sigma=+1$; and (c) $\rho_{T} / \sigma=-1, \rho_{B} / \sigma=+3$. 
In these examples, however, the vertical velocity decreases toward the bottom, while the magnitude of the meridional component increases to a maximum: horizontal advection must therefore dominate over vertical advection in the density balance at the bottom, and perhaps in the few hundred meters above the bottom as well, given the reduced magnitude of the vertical density gradient actually observed there. Furthermore, in the very deep South Pacific the overall pattern of horizontal density variation is a decrease northward toward the equator (e.g., Knauss, 1962). If, as suggested above, the deepest water is flowing equatorward, then the tendency of the meridional density advection would be to require negative curvature toward the bottom of the density-depth curve, as is observed (Fig. 1). To tell whether this effect is really significant in terms of observations, however, requires a much more elaborate solution of (1) than can be undertaken here, including nonlinear treatment of both the vertical and horizontal advections.

\section{Western boundary region}

The density balance in the region of the deep western boundary current is probably different from (2). The author has suggested elsewhere (Warren, 1976) that linear boundary layer theory for a stratified ocean may give a fairly good description of the structure of the deep western boundary current in the South Pacific. In that theory the boundary region is characterized by a perturbation to the interior density field that, although small in magnitude compared with the interior variation, is nevertheless of such small horizontal scale that it controls the boundary current dynamics-the control being through an approximate balance between vertical advection of the total density (in effect, the interior density) and lateral diffusion of the perturbation density.

To explore the relation between the motion field and the density-depth curve, consider a simplified version of that theory. Let the total density $\rho$ be given, in the spirit of the preceding section, as $\rho=-\Delta z / H+\rho^{*}$, where the first term is a representation of the interior field, $\rho^{*}$ is now the western boundary perturbation and $\rho^{*} \ll \Delta$. The approximate density balance is then

$$
K \rho_{x x}^{*}+w \Delta / H=0,
$$

where $w$ is the vertical velocity in the boundary region less the (much smaller) interior velocity.

Since viscosity is important to the property fields only very near the boundary (Warren, 1976), we disregard it here. The geostrophic, hydrostatic and geostrophic vorticity relations may then be combined with (7) to give

$$
\rho_{\rho z z x x}^{*}-\frac{\beta g \Delta}{K f^{2} \rho_{0} H} \rho_{x}^{*}=0
$$

and if solutions of the form $\rho^{*}=\rho^{\prime}(z) \exp (-\alpha x)$ are again sought, Eq. (8) becomes

$$
\rho_{z z}^{\prime}+\lambda^{2} \rho=0,
$$

where $\lambda^{2} \equiv(\beta g \Delta) /\left(K f^{2} \rho_{0} H \alpha\right)$.

We let the boundary conditions on (9) be $w=0$ at $z=0$ and $v=0$ at $z=H$. These are equivalent to $\rho^{\prime}(0)=0$, $\rho_{z}^{\prime}(H)=0$, and they determine eigenvalues for $\lambda$ (and therefore $\alpha$ ) with corresponding eigenfunctions for $\rho^{*}$ :

$$
\rho_{n}^{*}=Q_{n} e^{-\alpha_{n} x} \sin \lambda_{n} z
$$

where

$$
\lambda_{n}=\frac{(2 n+1) \pi}{2 H}, \quad \alpha_{n}=\frac{4 \beta g H \Delta}{(2 n+1)^{2} \pi^{2} K f^{2} \rho_{0}},
$$

where $n=0,1,2,3, \ldots$, and the $Q_{n}$ 's are constants chosen to fit the density-depth variation observed near the western boundary.

For an individual eigenfunction the meridional velocity $v_{n}$ is related to the vertical density gradient $\rho_{n z}^{*}$ by

$$
v_{n}=-\frac{f K H}{\beta \Delta} \alpha_{n}^{2} \rho_{n z}^{*}
$$

and $v_{n}$ is therefore zero at maxima or minima in $\rho_{n}^{\prime}$ and assumes its extreme values at the extrema in $\rho_{n z}^{\prime}$. The deep circulation is driven by global exchanges at the sea surface, however, and it seems likely in general that the density structure of the complete, deep western boundary flow would have to be represented by a sum of several eigenfunctions (10). In this case, because of the factor $(2 n+1)^{-4}$ in $\alpha_{n}^{2}$, which appears in (11), there would be no explicit relation across the full breadth of the flow between the sum of the $v_{n}$ 's and the sum of the $\rho_{n 2}^{*}$ 's.

Furthermore, although the velocity structure of the boundary region is determined essentially by $\rho^{*}(x, z)$, the latter is supposed to be only a small perturbation to the interior density field. Deviations of the latter from a strictly linear vertical variation, such as were calculated in the previous Section, would probably be comparable to the western boundary correction $\rho^{*}$, and hence the total density field in the western boundary region would be even further removed from any direct physical relation to the velocity field.

For a specific current field involving only two eigenfunctions, $Q_{0}=Q_{1}$, the depth of maximum perturbation density, depth of maximum perturbation stability (i.e., maximum value of $-\rho_{z}^{*}$ ), and depth where $v=0$ are compared in Fig. 5 in a zonal cross section of the current. The physical parameters are as in the preceding sections, with the addition that $K=0.5 \times 10^{7} \mathrm{~cm}^{2} \mathrm{~s}^{-1}$. The eigenfunction $n=0$ decays much more rapidly to the eastward than the other, and in the eastern part of the current, where only eigenfunction $n=1$ is significant, the depths where $\rho^{*}$ is maximum and $v=0$ coincide at a level about $1 \mathrm{~km}$ above the bottom. To the west, how- 


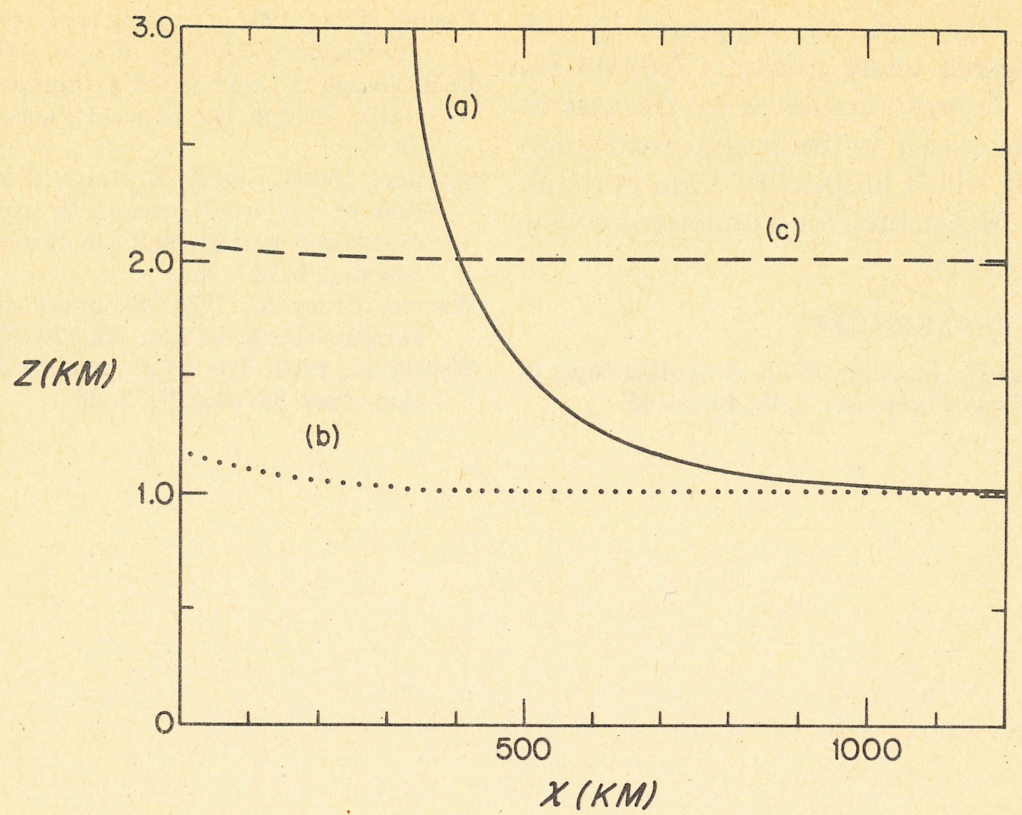

FIG. 5. Theoretical distributions across a deep western boundary current for which $Q_{0}=Q_{1}$, other $Q_{n}=0$, of the depths of (a) $v=0$, (b) maximum perturbation density and (c) maximum perturbation stability. Values of physical parameters given in text.

ever, where both eigenfunctions determine the structure, the two features diverge markedly, the surface where $v=0$ rising to the level $3 \mathrm{~km}$ above the bottom (the level is one where $v=0$ anyway). This surface bears no correspondence to that of maximum perturbation stability, which remains close to the level $z=2 \mathrm{~km}$ across the current.

Although the latter surface varies little in depth, one would expect the depth of maximum total stability to change substantially. Toward the eastern limit of the current, it should be determined essentially by the interior density field, which has a deep stability maximum, say, $1 \mathrm{~km}$ above the bottom (Fig. 1); but near the western boundary the perturbation field would also make an important contribution, and its stability maximum is at a considerably shallower level in Fig. 5.

The author has remarked (Warren, 1976) on the shortcomings of this theory for deep western boundary currents, especially that in the South Pacific it produces a zero surface for $v$ in the eastern part of the boundary region that is several hundred meters too deep; and I have suggested that this discrepancy may result from omitting the small meridional advection term from (7). Nevertheless, the theory as it stands seems to offer a useful first-order guide to the relation-or lack of itbetween the velocity field and the density-depth curves.

\section{Implications for interpreting observations}

In the examples for the interior region (Section 2), the curvature of the density-depth curve vanishes at the level of zero vertical velocity, and the meridional velocity vanishes at the level of maximum downward velocity, which is the level of maximum positive curvature. In more realistic calculations that included some horizontal advection and the full nonlinearity, or that at least involved linearization about a nonconstant basic density gradient, these correspondences would not be exact, but they nonetheless point to a tendency. And they do not support the opinion of Craig et al. (1972) that $v=0$ at the level of the upper inflection point (where $w=0$ ), nor the suggestion by Reid and Lonsdale (1974) that $v=0$ at the level of the lower inflection point (maximum stability).

In the western boundary theory, it is not the curve of total density against depth which gives information about the velocity field, but the curve with the interior density variation subtracted out. Maxima or minima in perturbation density (not perturbation stability) then specify levels where $v=0$, but only if the perturbation density structure is dominated by a single eigenfunction of (8). In the more probable situation that the structure represents a combination of eigenfunctions, a surface where $v=0$ cannot be identified across the region with any feature of the density distribution. Probably inflections, or maxima or minima in perturbation density or total stability, can always be mapped across deep western boundary currents, but they will not have any necessary relation to the velocity field.

It might be noted that the difficulty with combined eigenfunctions does not pertain to the interior region. For any solution of the form $\rho^{*}(x, z)=\rho^{\prime}(z) \exp (-\alpha x)$, $v \propto \rho_{z z z}^{*}$ there, and for any sum of such solutions, each specified by a different value of $\alpha$, the same proportionality holds. 
Acknowledgments. This work was supported by the Office of Naval Research under Contract N00014-74CO262 NR 083-004. It was stimulated by discussions with J. L. Reid during a visit to the Scripps Institution of Oceanography, for which hospitality I am grateful. I am indebted to M. McCartney for a prolonged review of a first draft of this paper.

\section{REFERENCES}

Craig, H., Y. Chung and M. Fiadeiro, 1972: A benthic front in the South Pacific. Earth Planet. Sci. Lelt., 16, 50-65.
Knauss, J. A., 1962: On some aspects of the deep circulation of the Pacific. J. Geophys. Res., 67, 3943-3954.

Reid, Joseph L., and Peter F. Lonsdale, 1974: On the flow of water through the Samoan Passage. J. Phys. Oceanogr., 4, 58-73.

Stommel, Henry, and A. B. Arons, 1960 : On the abyssal circulation of the world ocean-II. An idealized model of the circulation pattern and amplitude in oceanic basins. Deep Sea Res., 6, 217-233.

Warren, Bruce A., 1976: Structure of deep western boundary currents. Deep Sea Res., 23, 129-141.

Wyrtki, K., 1962 : The oxygen minima in relation to ocean circulation. Deep Sea Res., 9, 11-23. 


\section{MANDATORY DISTRIBUTION LIST \\ FOR UNCLASSIFIED TECHNICAL REPORTS, REPRINTS, \& FINAL REPORTS PUBLISHED BY OCEANOGRAPHIC CONTRACTORS \\ OF THE OCEAN SCIENCE AND TECHNOLOGY DIVISION OF THE OFFICE OF NAVAL RESEARCH}

(REVISED FEBRUARY 1978)

1 Director of Defense Research and Engineering

Office of the Secretary of Defense Washington, D.C. 20301

ATTN: Office Assistant Director (Research)

Office of Naval Research Arlington, VA 22217

1 ATTN: (Code 460)

1 ATTN: (Code 102-OS)

6 ATTN: (Code 102IP)

1 ATTN: (Code 200)

1 CDR J. C. Harlett, (USN) ONR Representative Woods Hole Oceanographic Inst. Woods Hole, MA 02543

1 Office of Naval Research Branch office 495 Summer Street Boston, MA 02210

Director Naval Research Laboratory Washington, D.C. 20375

6 ATTN: Library, Code 2620
1 National Oceanographic Data Center National Oceanic \& Atmospheric Administration 3300 Whitehaven St., N.W. Washington, D.C. 20235

12 Defense Documentation Center

Cameron Station

Alexandria, VA 22314

Commander

Naval Oceanographic Office

Washington, D.C. 20373

1 ATTN: Code 1640

1 ATTN: Code 70

3 NORDA 430 NSTL Station, MS 39529

2 CO NORDA NSTL Station, MS 39529 



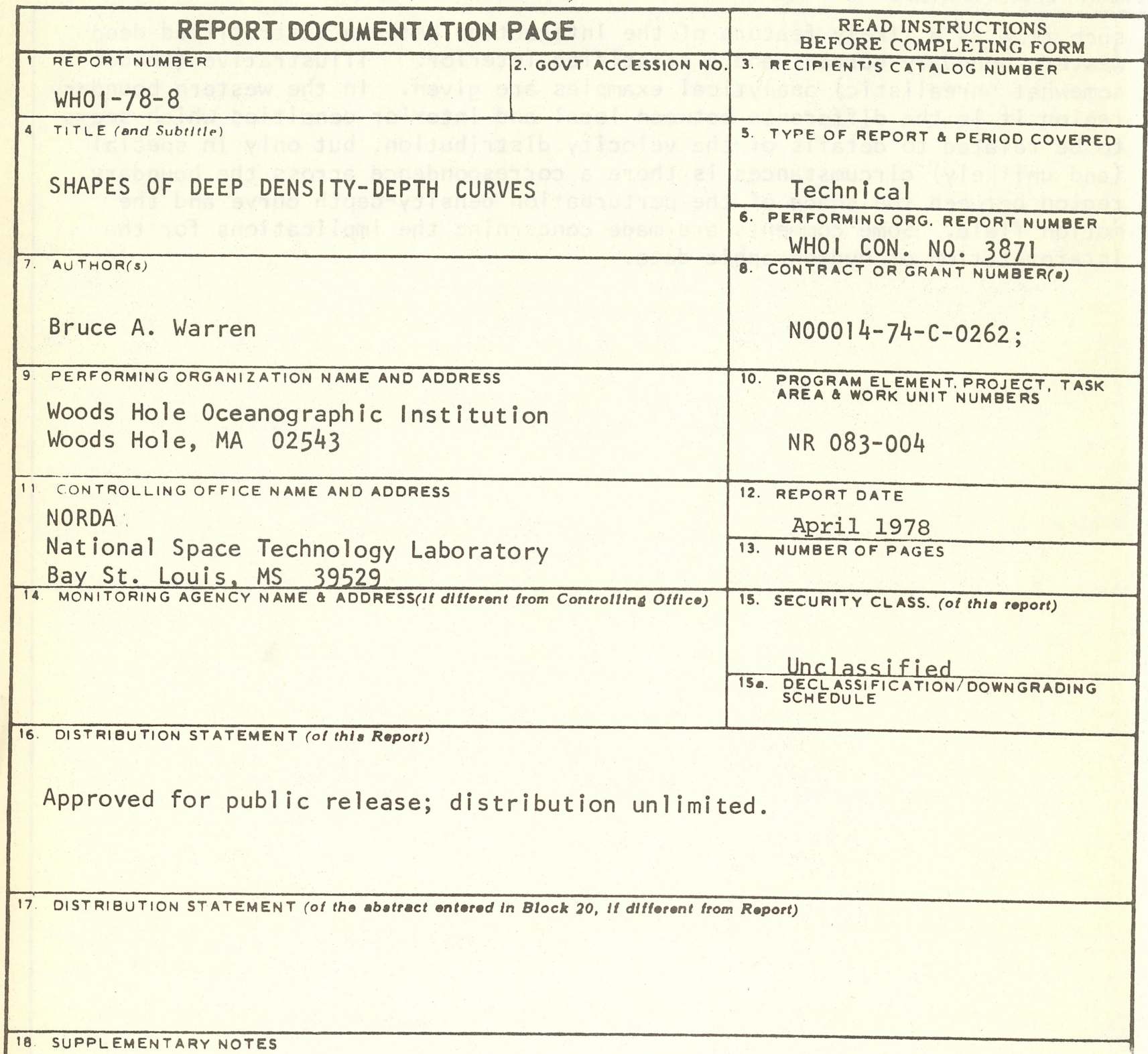

Reprinted from the "Journal of Physical Oceanography, Vol. 7, No. 3 May, 1977, pp. 338-344".

19. KEY WORDS (Continue on poverse alde if neceaseary and identlfy by block number)

1. South Pacific

2. Density-depth curves

3. Advection-diffusion

20. ABSTRACT (Continue on soverse olde if neceseary and ldentlfy by block number)

Deep density-depth curves often show faint curvature reversals, stability maxima, etc. Relationships between these features and the field of motion are explored here, in reference to the South Pacific, for interior regions and western boundary currents. For the former, an assumed balance between vertical advection and vertical diffusion of density implies downward movement where there is positive curvature in the density-depth curve, in association with equatorward flow of the bottom water; it is argued that (Cont. on back)

DD, FORM 1473 EDITION OF I NOV 63 IS OBSOLETE


such flow is a likely feature of the interaction between a stratified deep western boundary current and a stratified interior. Illustrative (though somewhat unrealistic) analytical examples are given. In the western boundary region it is the difference between local and interior densities which seems to be related to details of the velocity distribution, but only in special (and unlikely) circumstances is there a correspondence across the boundary region between the shape of the perturbation density-depth curve and the motion field. Some comments are made concerning the implications for the interpretation of hydrographic data. 


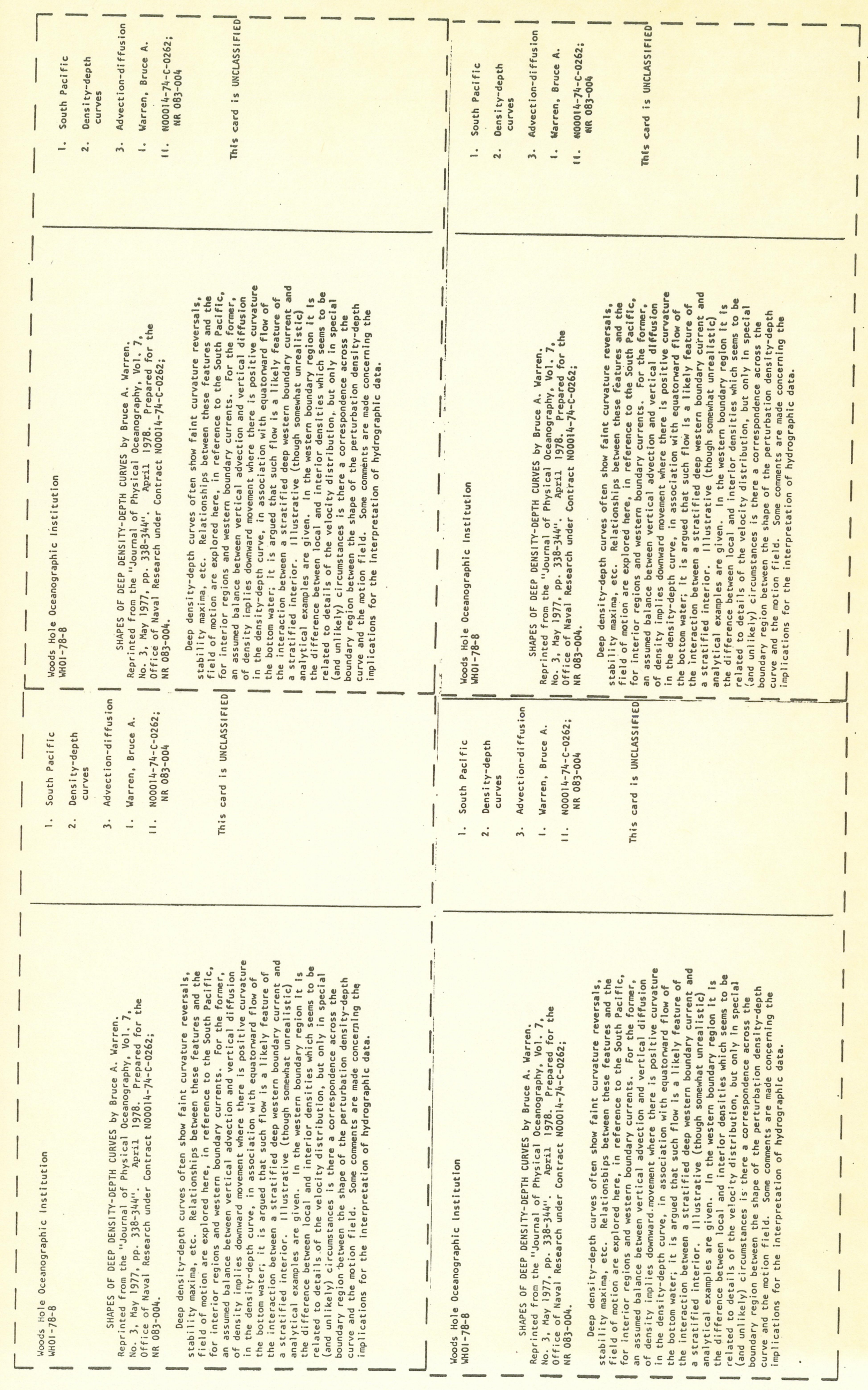


